\title{
Les médecins valaisans lancent un référendum pour une politique sanitaire transparente incluant les professionnels de la santé
}

\section{Monique Lehky Hagen}

Présidente du Comité Référendaire LeisReferendum
Correspondance: Dr Monique Lehky Hagen FMH Médécine Interne Case Postale 596 CH-3900 Brig

leisreferendum@netplus.ch www.leisreferendum.ch
Suite à l'introduction des tarifs DRG dès 2012 dans toute la Suisse, les lois cantonales doivent être adaptées à ce changement. Dans cette optique, la loi sur les établissements et institutions sanitaires (LEIS) a été révisée en Valais. Par la même occasion, les articles concernant la planification hospitalière auraient pu être révisées afin d'améliorer certains dysfonctionnements. Malheureusement, le Grand Conseil Valaisan n'a pas tenu compte des prises de position de la Société Médicale Valaisanne, ni de différentes interventions du corps médical - entre autre d'une lettre ouverte au Grand Conseil, signée par plus de 200 médecins qui demandaient le report de la ratification de cette loi. Suite à cela, un comité regroupant presque 40 médecins s'est constitué et a lancé un référendum contre cette révision de loi.

Dans le Canton du Valais, la minorité germanophone Haut-Valaisanne rencontre de grands problèmes linguistiques lors d'hospitalisations à l'Hôpital de Sion, où les disciplines spécialisées ont été centralisées (neurochirurgie, chirurgie cardiaque, thoracique, cardiologie invasive, radio-oncologie...). Les patients qui n'ont que l'assurance de base, n'ont pas d'autre alternative. Cela ne changera pas en 2012, malgré le soi-disant «libre-choix des Hôpitaux», à cause du point tarifaire bas en Valais. Une communication correcte entre le patient, sa famille et le corps médical et soignant n'est pas garantie. Aucun effort particulier n'a été entrepris pour améliorer cette situation ces 10 dernières années. Ceci est d'autant plus étonnant qu'un service téléphonique national vient d'être mis sur pieds par le programme national migration et santé qui propose des traductions médicales $24 \mathrm{~h} / 24$ pour les migrants dans 12 langues (hormis les langues nationales)! Ceci devrait illustrer assez clairement l'importance du problème.

En ce qui concerne la planification hospitalière, le corps médical n'a pratiquement rien à dire depuis des années et doit se contenter d'exécuter la planification décidée par les politiques.

Ceci explique aussi, pourquoi les exigences claires formulées par les médecins ont pu être ignorées et que même une lettre ouverte au Conseil d'Etat est restée sans réponse.

Suite à différents scandales dans le milieu hospitalier Valaisan en 2010, le Grand Conseil a décidé contre l'avis du Conseil d'Etat de demander un audit sur le «ré- seau santé valais» (RSV, «Hôpital Valais»). Cet audit a duré 7 mois et a coûté plus d'un million de francs aux contribuables.

Dans cet audit, d'importants problèmes sont retenus dans la structure administrative et dans la gestion du personnel du RSV. Pour résoudre certains de ces problèmes, des changements de certains articles figurants dans la LEIS sont incontournables. Le Grand Conseil avait refusé de repousser le vote sur la loi révisée comme l'avaient exigé les médecins, alors qu'il était connu que les résultats de l'audit étaient sur le point d'être publiés. Dans le même laps de temps, les résultats du rapport de la Commission de Gestion du Grand Conseil (Cogest) furent eux aussi présentés, arrivant à des résultats similaires.

Après des discussions avec tous les partenaires y compris les présidents Haut-Valaisans (resp. leur remplaçant) de tous les partis nous avons conclus qu'il était indispensable de lancer le référendum contre cette loi pour des raisons éthiques et de politique professionnelle.

Nous exigeons:

- l'ancrage du bilinguisme pour les disciplines centralisées et déléguées;

- l'intégration des arguments des professionnels de la santé dans la LEIS;

- l'intégration des conclusions du rapport de l'audit

+ Cogest dans la LEIS.

Nous sommes tenus à nous engager pour garantir des soins médicaux optimaux pour la population valaisanne. Dans ce sens, il nous faut exiger une politique sanitaire transparente et basée sur des faits.

La situation actuelle devrait nous faire réfléchir sur le nadir que nous avons atteint du point de vue de notre politique professionnelle...

Nous comptons sur un engagement important du corps médical et de la population, afin de pouvoir reconquérir le droit d'être entendu et d'être pris au sérieux, afin de pouvoir assumer le rôle déterminant qui devrait nous revenir dans une politique de santé au service de nos patients en assurant une collaboration optimale entre les différents intervenants de notre système de santé.

Vous trouverez plus d'informations sous: www. leisreferendum.ch 